\title{
Prevalência de Tabagismo entre Escolares em Município de Área Metropolitana da Região Sul, Brasil, 1991
}

\section{Smoking Prevalence among Students from a Metropolitan Area in the Southern Region of Brazil, 1991}

\author{
Ronaldo Bordin ${ }^{1}$; Vera B. Nipper ${ }^{2}$ \\ Jacqueline O. Silva ${ }^{1}$ \& Luciana Bortolomiol ${ }^{1}$
}

BORDIN, R.; NIPPER, V. B.; SILVA, J. O. \& BORTOLOMIOL, L. Smoking Prevalence among Students from a Metropolitan Area in the Southern Region of Brazil, 1991. Cad. Saúde Públ., Rio de Janeiro, 9 (2): 185-189, Apr/Jun, 1993.

A prevalence study on smoking habits was carried out in 1991, among 864 school children from eight municipal schools in Sapiranga, in the State of Rio Grande do Sul, Brazil, from the 6th through 8th grade of primary school. Data was collected in class, using a questionnaire applyed by trained teachers. Among students, 3.2\% were smokers, although $20.3 \%$ had been exposed to tobacco. Males had begun the smoking habit at a mean age of 14.7 years and females at about 15 years old. The prevalence showed an increasing trend by age and individual school grade. As for gender, male smokers outnumbered females $(p<0,05)$. Smoking by older brothers and fathers seems to be positively associated with acquisition of the habit by school children.

Key words: Smoking; Cross Sectional; Students

\section{INTRODUÇÃO}

O tabaco é a droga mais comumente usada e amplamente disseminada no mundo atual, sendo responsável por 5\% da mortalidade geral mundial. Pouco mais de 50 mil trabalhos comprovam seus efeitos deletérios (Rosemberg, 1987), incluindo em seu bojo estudos prospectivos com décadas de duração, como o realizado em 34 mil médicos britânicos (Doll \& Hill, 1964; Doll \& Peto, 1976), já com mais de 25 anos de acompanhamento, e o patrocinado pela American Cancer Society, com pouco mais de um milhão de pessoas e treze anos de seguimento (Hammond, 1966).

Apenas como exemplo, o tabagismo é responsável por $80 \%$ dos casos de enfisema pulmonar,

\footnotetext{
${ }^{1}$ Departamento de Medicina Social da Faculdade de Medicina da Universidade Federal do Rio Grande do Sul. Rua Ramiro Barcelos, 2600 - Térreo. Porto Alegre, RS, 90035-003, Brasil.

${ }^{2}$ Rua Otto Kunz, 176/14. Sapiranga, RS, 93800-000, Brasil.
}

$80 \%$ dos casos de câncer de pulmão, $75 \%$ dos casos de bronquite crônica e $25 \%$ dos casos de infarto do miocárdio (Hijjar \& Silva, 1991). No Brasil, há estimativas de que devam morrer, anualmente, em torno de 80 a 100 mil pessoas em decorrência de doenças relacionadas ao tabaco (Barbosa et al., 1989; Hijjar \& Silva, 1991). Um quadro sintético quanto aos agravos provocados pelo uso do tabaco pode ser encontrado em Hirayama (1987).

Um dos enfoques privilegiados nas pesquisas brasileiras é o estudo da prevalência deste hábito na população em geral ou em grupos específicos, como gestantes (Madi et al., 1984; Simões, 1985; Siqueira et al., 1985) escolares (Barbosa et al., 1989; Carvalho, 1987; Hijjar \& Silva, 1991), estudantes de medicina (Gadia et al., 1981; Horta et al., 1988; Rosemberg \& Peron, 1990; Szego et al., 1985) e trabalhadores (Hijjar \& Silva, 1991).

Estudos nacionais realizados entre escolares apontam uma prevalência de tabagismo que varia de 4 a $20 \%$ ou mais, quando considerados estudantes de $1^{\circ}$ e $2^{\circ}$ graus, chegando até $30,5 \%$, em amostras compostas apenas por 
estudantes de nível secundário (Barbosa et al., 1989; Hijjar \& Silva, 1991). Levantamento realizado no período de 1980 a 1984 em escolares de $1^{\circ}$ e $2^{\circ}$ graus de Porto Alegre constatou uma prevalência média do hábito de fumar de $12,8 \%$ e $10,5 \%$, respectivamente (Hijjar \& Silva, 1991). Ainda entre escolares, vários estudos já demonstraram que há um aumento progressivo de fumantes com a idade e com o ano escolar (Hijjar \& Silva, 1991). Além disso, o uso de tabaco está positivamente associado ao fato de o estudante trabalhar, freqüentar curso noturno, estar atrasado nos estudos e ter pais fumantes (Barbosa et al., 1989).

O objetivo deste estudo é descrever a prevalência do uso de tabaco entre escolares da $6^{\mathrm{a}}$ à $8^{\mathrm{a}}$ série do $1^{\mathrm{o}}$ grau da rede municipal de ensino de Sapiranga, Rio Grande do Sul, em 1991.

\section{MATERIAL E MÉTODOS}

O presente estudo foi realizado na rede pública municipal de ensino de Sapiranga, município integrante da região metropolitana de Porto Alegre (RS), contando com 58.387 habitantes (Fibge, 1992) e com economia centrada na indústria calçadista, notadamente de caráter exportador. A rede pública municipal de ensino é composta por dezenove escolas, todas diurnas. O critério mínimo de inclusão das escolas no estudo foi possuírem ao menos uma turma de sexta série do $1^{\circ}$ grau, o que reduziu o número de escolas a nove. Assim, a população do estudo corresponde à totalidade dos alunos presentes $(\mathrm{n}=864)$ no dia de aplicação do questionário, respondido de forma voluntária e anônima, em oito das nove escolas públicas municipais.

Uma escola com apenas uma turma de $6^{\mathrm{a}}$ série, embora tenha aplicado os questionários, não entrou neste estudo por problemas administrativos. A soma destes alunos com os ausentes no dia de aplicação do questionário (por qualquer motivo, inclusive evasão) não atingiu 5\% do total de vagas ocupadas da $6^{\mathrm{a}}$ à $8^{\mathrm{a}}$ série. Não houve tentativa de resgate dos alunos faltosos apenas no dia de aplicação do questionário.

$\mathrm{O}$ instrumento empregado para a coleta de dados buscava descrever os estudantes segundo sexo, idade, contato prévio com o tabaco, categoria de tabagismo, data de início do hábito, frequiência, quantidade e existência ou não de familiar fumante.

Os questionários foram aplicados e recolhidos pelas professoras das respectivas séries, previamente treinadas por um dos autores. Os dados foram codificados, tabulados e analisados no Departamento de Medicina Social da Faculdade de Medicina, Universidade Federal do Rio Grande do Sul (UFRGS). Empregou-se o teste do qui-quadrado e o "t" de Student nas análises, para um alfa de $5 \%$.

Esta atividade compunha a programação da Semana Municipal de Combate ao Fumo, agregada ao Dia Mundial de Combate ao Fumo, estando a mesma integrada ao projeto "A Realidade Atual de Sapiranga: uma proposta de pesquisa-ação em busca de um planejamento participativo" (com fomento da Fundação de Amparo à Pesquisa do Estado do Rio Grande do Sul - Fapergs).

Para este estudo, a categoria "fumante" foi operacionalizada como sendo os indivíduos que relataram ter fumado ao menos um (1) cigarro/dia, por um período mínimo de seis meses. Ex-fumante correspondia ao fumante que havia abandonado o hábito há pelo menos seis meses, respeitada a caracterização de fumante. A categoria familiar consistia em membros próximos (pai, mãe e irmãos) ou outro membro da família extensa, desde que residente no mesmo domicílio.

\section{RESULTADOS}

Um total de 864 alunos respondeu ao questionário. Destes, 434 (50,2\%) eram do sexo masculino e $420(48,6 \%)$, do sexo feminino, sendo que $10(1,2 \%)$ alunos não responderam a esta questão. Não houve diferença estatisticamente significativa com relação ao sexo e à série escolar.

A idade média foi de $14,69 \pm 2,43$ anos, variando de 11 a 30 anos, com $90 \%$ dos casos entre 12 e 18 anos. A mediana ficou em 14 anos. A idade média e a mediana masculinas foram de 15 anos e as femininas, de 14 anos. A 
explicação para a idade média elevada nas séries estudadas deve levar em conta uma particularidade municipal: a maior parte das crianças em idade escolar frequenta as escolas municipais até a $4^{\mathrm{a}}$ série do $1^{\circ}$ grau, então, quando são incorporadas à indústria calçadista. No momento, existe um forte estímulo por parte do empresariado e do executivo municipal para que os adultos (15 anos ou mais) retornem aos bancos escolares, já que um estudo realizado pela Prefeitura Municipal de Sapiranga identificou analfabetismo, dificuldades de leitura e/ou de redação notadamente nestas faixas etárias.

A Tabela 1 apresenta a distribuição do número de alunos por série e categoria de tabagismo. Encontrou-se uma prevalência global de 3,2\%, com predominância do sexo masculino - 21 (75\% dos casos) ( $\mathrm{p}<0,05)$. Estes dados vão de encontro à literatura, que mostra uma baixa, mas preocupante, prevalência entre jovens escolares (Barbosa et al., 1989; Hijjar \& Silva, 1991). Ressalvas quanto à qualidade desta informação devem ser consideradas, já que a aplicação do questionário por parte dos professores pode ter influenciado na qualidade da resposta, diminuindo o número de fumantes e superestimando o número de não-fumantes, mas que apresentaram contato com o hábito.
TABELA 1. Número de Estudantes por Série e Percentual de Fumantes

\begin{tabular}{lcccc}
\hline \hline \multirow{2}{*}{$\begin{array}{l}\text { Série } \\
\text { Escolar }\end{array}$} & \multicolumn{2}{c}{ Não-Fumantes } & \multicolumn{2}{c}{ Fumantes } \\
\cline { 2 - 5 } & $\mathrm{n}$ & $\%$ & $\mathrm{n}$ & $\%$ \\
\hline $6^{\mathrm{a}}$ & 426 & 50,9 & 14 & 3,2 \\
$7^{\mathrm{a}}$ & 264 & 31,3 & 06 & 2,2 \\
$8^{\mathrm{a}}$ & 146 & 17,8 & 08 & 5,2 \\
\hline Total & 838 & 100,0 & 28 & 3,2 \\
\hline \hline
\end{tabular}

A idade de início do hábito apresentou sua mediana aos 15 anos, com uma média de 14,7 e 15 anos para os sexos masculino e feminino, respectivamente. A quantidade média de cigarrros fumados por dia foi de 13,8 entre os homens e de 9,7 entre as mulheres.

Embora apenas $28(3,2 \%)$ alunos fossem fumantes, $175(20,3 \%)$ referiram consumir tabaco ao menos uma vez, atingindo $23,5 \%$ dos meninos e $16,4 \%$ das meninas de todas as séries (Tabela 2).

$\mathrm{Na}$ Tabela 3 encontra-se a prevalência de familiar fumante entre os alunos fumantes e não-fumantes. Entre os escolares fumantes havia um familiar fumante em 23 (82\%) dos 28 casos, magnitude percentual que reduz-se a $10 \%$ entre os escolares não-fumantes $(\mathrm{p}<0,05)$.

TABELA 2. Número e Percentual de Escolares Fumantes e que Apresentaram Contato com Tabaco, Sistematizados por Sexo e Série Escolar

\begin{tabular}{|c|c|c|c|c|c|c|c|c|}
\hline \multirow{3}{*}{$\begin{array}{l}\text { Série } \\
\text { Escolar }\end{array}$} & \multicolumn{4}{|c|}{ Contato $^{(1)}$} & \multicolumn{4}{|c|}{ Fumante ${ }^{(2)}$} \\
\hline & \multicolumn{2}{|c|}{ Masc. } & \multicolumn{2}{|c|}{ Fem. } & \multicolumn{2}{|c|}{ Masc. } & \multicolumn{2}{|c|}{ Fem. } \\
\hline & $\mathrm{n}$ & $\%$ & $\mathrm{n}$ & $\%$ & $\mathrm{n}$ & $\%$ & $\mathrm{n}$ & $\%$ \\
\hline $6^{\mathrm{a}}$ & 47 & 46,1 & 30 & 43,5 & 11 & 52,4 & 3 & 42,9 \\
\hline $7^{\mathrm{a}}$ & 34 & 33,3 & 26 & 37,7 & 04 & 19,0 & 2 & 28,6 \\
\hline $8^{a}$ & 21 & 20,6 & 13 & 18,8 & 06 & 28,6 & 2 & 28,6 \\
\hline Total & 102 & 59,6 & 69 & 40,4 & 21 & 75,0 & 7 & 25,0 \\
\hline
\end{tabular}

(1) Diferença não-significativa.

(2) $\mathrm{p}<0,05$. 
TA BELA 3. Percentual de Familiar Fumante entre Escolares, Fumantes ou Não

\begin{tabular}{|c|c|c|c|c|}
\hline \multirow[b]{2}{*}{$\begin{array}{l}\text { Familiar } \\
\text { Fumante }\end{array}$} & \multicolumn{2}{|c|}{ Escolar Fumante } & \multicolumn{2}{|c|}{ Escolar Não-Fumante } \\
\hline & $\mathrm{n}$ & $\%$ & $\mathrm{n}$ & $\%$ \\
\hline Pai & 4 & 14,3 & 31 & 3,8 \\
\hline Mãe & 2 & 7,1 & 8 & 0,9 \\
\hline Irmãos & 4 & 14,3 & 19 & 2,3 \\
\hline Pai + mãe & 3 & 10,7 & 17 & 2,1 \\
\hline Pai + irmãos & 6 & 21,4 & 8 & 0,9 \\
\hline Mãe + irmãos & 1 & 3,6 & 3 & 0,4 \\
\hline Outros familiares & 3 & 10,7 & 10 & 1,2 \\
\hline Nenhum familiar & 5 & 17,9 & 740 & 89,6 \\
\hline Total & 28 & 3,2 & 836 & 96,8 \\
\hline
\end{tabular}

\section{CONCLUSÃO}

O presente estudo revelou uma prevalência para o hábito de fumar de $3,2 \%$, com predominância do sexo masculino ( $75 \%$ dos casos fumantes). Ao mesmo tempo, o ambiente familiar parece exercer forte influência, já que $83 \%$ dos escolares classificados como fumantes conviviam com pelo menos um familiar fumante. Os exemplos paterno e fraterno constituiramse num forte estímulo para a adesão ao hábito de fumar.

Apesar dos resultados referendarem trabalhos anteriormente realizados, um saldo positivo deve ser agregado a este estudo: ele propiciou um incremento na discussão entre a comunidade escolar do tabagismo, sendo complementada por atividades paralelas, tais como a estruturação de um programa municipal de combate ao fumo, a institucionalização de uma Semana Municipal de Combate ao Fumo, incluindo nesta o Dia Mundial de Combate ao Fumo, e um concurso entre escolares de primeiro grau, cujos trabalhos vencedores (escolhidos pelos próprios alunos) se constituem nas estampas de cartazes distribuídos a todas escolas e à comunidade em geral alertando quanto aos malefícios do fumo.

\section{RESUMO}

BORDIN, R.; NIPPER, V. B.; SILVA, J. O. \& BORTOLOMIOL, L. Prevalência de Tabagismo entre Escolares em Município de Área Metropolitana da Região Sul, Brasil, 1991. Cad. Saúde Públ., Rio de Janeiro, 9 (2): 185-189, abr/jun, 1993.

Em 1991, foi realizado um estudo de prevalência sobre o hábito de fumar entre 864 alunos da $6^{\mathrm{a}}$ à $8^{\mathrm{a}}$ série do $1^{\mathrm{o}}$ grau de oito escolas municipais de Sapiranga, Rio Grande do Sul. A coleta de dados foi feita através de um questionário aplicado e recolhido por professoras treinadas, em sala de aula. Entre os escolares, $3,2 \%$ eram fumantes, embora $20,3 \%$ tenham sido expostos pelo menos uma vez ao tabaco. A idade média de início do hábito foi de 14,7 anos no sexo masculino e 15,0 no sexo feminino. A prevalência mostrou uma tendência a aumentar junto com a idade e com o ano escolar. Quanto ao sexo, o número de fumantes foi significativamente maior no sexo masculino ( $\mathrm{p}<0,05)$. O hábito de fumar do irmão mais velho e do pai parece reforçar a adesão do escolar ao mesmo.

Palavras-Chave: Fumo; Estudos de Prevalência; Estudantes 


\section{REFERÊNCIAS BIBLIOGRÁFICAS}

BARBOSA, M. T. S.; CARLINI-COTRIM, B. \& SILVA-FILHO, A. R., 1989. O uso de tabaco por estudantes de primeiro e segundo graus de dez capitais brasileiras: possíveis contribuições de estatística multivariada para a compreensão do fenômeno. Revista de Saúde Pública, 23: 401-409.

CARVALHO, F. M., 1987. Hábito de fumar em adolescentes escolares de Salvador, Bahia. Revista Baiana de Saúde Pública, 14: 212-216.

DOLL, H. \& HILL, A. B., 1964. Mortality in relation to smoking: ten years' observation of British doctors. British M edical Journal, 1: 1399.

DOLL, H. \& PETO, R., 1976. Mortality in relation to smoking: 20 years' observation on male British doctors. British M edical J ournal, 2: 1525.

GADIA, C.; SALTZ, R.; BARTCZAK, M. \& GONZALES, M. C., 1981. O estudante de medicina e o fumo. Revista da Associação M édica do Rio Grande do Sul, 25: 8-13.

HIJJAR, M. A. \& SILVA, V. L. C., 1991. Epidemiologia do tabagismo no Brasil. Jornal Brasileiro de M edicina, 60: 50-71.

HIRAYAMA, T., 1987. Health effects of active and passive smoking - smoking health. Excerpta M édica, 75-86, ICS 780.

HORTA, B. L.; RAMOS, E. O.; VICTORA, C. G., 1988. O hábito de fumar entre estudantes de medicina da UFPEL: prevalência, sintomatologia respiratória e relação com o tabagismo dos pais. Revista da Associação M édica do Rio Grande de Sul, 32: 15-17.
MADDI, J. M.; COELHO, C. P. \& LUNARDI, P., 1984. O uso de fumo durante o ciclo gravídico. Considerações fisiopatológicas e correlação preliminar com população de 1559 pacientes. Jornal Brasileiro de Medicina, 46: 42-45.

ROSEMBERG, J., 1987. Tabagismo e Saúde. Informação para Profissionais de Saúde. Brasília: Centro de Documentação do Ministério da Saúde.

ROSEMBERG, J. \& PERON, S., 1990. Tabagismo entre estudantes da Faculdade de Ciências Médicas de Sorocaba. Tabagismo nos acadêmicos de medicina e nos médicos. Jornal de Pneumologia, 16: 13-22.

SIMÕES, M. J. S., 1985. Estudo da freqüência do hábito de fumar durante a gestação. Ribeirão Preto - SP. Revista de Ciências Biomédicas, 6: 61-69.

SIQUEIRA, A. A. F.; SANTOS, J. L. F.; SAQUETO, C. G.; LUZ, E. T. \& ARAÚJO, M. C. A., 1985. Estado nutricional e hábito de fumar maternos, crescimento intra-uterino e pós-natal. Revista de Saúde Pública, 19: 37-50.

SZEGO, T.; BECHARA, M. J. \& GAMA-RODRIGUES, J., 1985. Levantamento epidemiológico sobre o vício de fumar entre estudantes de medicina no estado de São Paulo. Revista da A ssociação M édica Brasileira, 31: 13-16. 